\title{
Computational Model for Simulating Drug-Induced Arrhythmia Sensitivity of Human iPSC-derived Cardiomyocytes
}

\author{
Xin $\mathrm{Gao}^{2}$, Yue Yin ${ }^{1}$, Neil Daily ${ }^{1}$, Tyler Engel ${ }^{1}$, Li Pang ${ }^{3}$, Brian E. Carlson ${ }^{2}$ and Tetsuro Wa- \\ katsuki $^{1}$ \\ ${ }^{1}$ InvivoSciences, Inc., Madison, WI \\ ${ }^{2}$ Department of Molecular and Integrative Physiology, University of Michigan, Ann Arbor, MI \\ ${ }^{3}$ Division of Biochemical Toxicology, National Center for Toxicological Research/FDA, Jeffer- \\ son, AR
}

\begin{abstract}
A mathematical model describing the electrophysiology and ion handling of cardiomyocytes is a complement to experimental analysis predicting drug-induced proarrhythmic potential in humans as proposed by Comprehensive in vitro Proarrhythmia Assay (CiPA). While CiPA endorses the use of the O'Hara Rudy (ORd) model, which was developed to simulate electrophysiology of human adult ventricular cardiomyocytes (hAVCMs), to predict drug-induced proarrhythmias; the human induced pluripotent stem cell derived- cardiomyocytes (hiPSC-CMs) was proposed for experimental verifications. The hiPSC-CMs, especially cultured in $2 \mathrm{D}$ culture dishes, are inherently different from hAVCMs exhibiting different ion channel density and an immature sarcoplasmic reticulum function. To reconcile this mismatch, we have developed a mathematical electrophysiology model of an hiPSC-CM by incorporating differences in gene expressions of ion channels, pumps and receptors in hiPSC-CMs against those found in hAVCMs. This model can be used to model any hiPSC-CM cell line where expression data has been obtained and replaces the background currents for $\mathrm{K}^{+}$and $\mathrm{Na}^{+}$in the ORd model with the known ultra-rapid $\mathrm{K}^{+}$channel and hyperpolarization activated $\mathrm{K}^{+} / \mathrm{Na}^{+}$channel currents. With this new model, three batches each from two different hiPSC-CM cell lines are compared to experimental data of action potential duration. This mathematical model recapitulates a ventricular-like action potential morphology with a Phase 2 plateau lasting a few hundred milliseconds. However, the resting membrane potential is not as depolarized $(-65$ to $-70 \mathrm{mV})$ as that of hAVCM $(-80 \mathrm{mV})$. The elevated resting membrane potential matches experimental data previously and is thought to keep rapid sodium channels from triggering membrane depolarization instead being replaced by an overexpression of L-Type $\mathrm{Ca}^{2+}$ channel current. This model is a key step in identifying the variability between different hiPSC-CM lines and even batches of the same line, opening the door to realizing analysis of patient specific preparations in the future.
\end{abstract}




\section{Introduction}

Over 130,000 ( 1/3 of total cardiac adverse events) cases of drug induced, potentially life-threatening, cardiac arrhythmias, torsades de points (TdP), were recorded by the US Food and Drug Administration (FDA) between 1969 and 2000 ${ }^{1}$. Some of these drugs were withdrawn from the market due to torsadogenic risk ${ }^{2}$. After the International Conference on Harmonization (ICH) issued ICHS7B and E14 Guidelines in $2005^{3}$, the rate of TdP reports has declined ${ }^{4}$. The S7B guideline recommends performing a simple hERG channel inhibition assay using cell lines (e.g., CHO or HEK cells) expressing the hERG channel as the primary in vitro assay to evaluate TdP risk. Despite its well-recognized role in assessing drug-induced QTc prolongation risk, solely based on hERG potency and therapeutic plasma exposure can result in incorrected conclusions and unwanted compound attrition during drug discovery ${ }^{5}$.

The Comprehensive in vitro Proarrhythmia Assay (CiPA) Initiative has defined a new paradigm by which computational models are identified with experimental data generated with heterologous systems to predict drug-induced proarrhythmia risk in human ventricular myocytes. These proposed theoretical models incorporate current density or flux and dynamic gating function of the major ion channels, pumps and receptors expressed in CMs. In coupling with detailed experimental data generated with hiPSC-CMs, the new in vitro assay systems can provide a more comprehensive view of cardiomyocyte function than that relying simply on compound-effects of hERG channel inhibition ${ }^{6}$. hiPSC-CMs express the same ion channels, pumps and receptors known to be expressed in human adult ventricular cardiomyocytes (hAVCMs) however the level of expression has been observed to be different ${ }^{7}$. hiPSC-CMs also exhibit action potentials (AP) profiles similar in form to hAVCMs but with a large variation in action potential duration at $90 \%$ repolarization $\left(\mathrm{APD}_{90}\right.$ ) of $70-789 \mathrm{~ms}^{8}$ and spontaneous beating frequencies of $25-85$ beats per minute ${ }^{7}$.

While hiPSC-CM derived tissues are a logical choice to improve the guideline ${ }^{9}$, their immaturity and under-developed excitation-contraction (EC) coupling ${ }^{10,11}$ are also of critical concern. A previous theoretical model of hiPSC-CMs electrophysiology and ion handling has been developed ${ }^{12}$ based on experimental data of ion channel function in hiPSC-CMs at 30-53 days post differentiation $^{13}$. While the previous model by Paci et al. is extremely successful in describing purified hiPSC-CMs at the 30-53 day maturation time point, a patch clamp study similar to that performed by Ma et al. would be needed to characterize other maturation time points or variation across different induced pluripotent stem cell lines. Therefore, we took a different approach using the latest hAVCM theoretical model by O'Hara et al. ${ }^{14}$ (ORd) coupled with relative expression data between hAVCMs and hiPSC-CMs for 11 genes associated with ion channel, pump, receptor and transporter density to determine the change of ionic transport driving electrophysiological function in an hiPSC-CM. In this new model, we have replaced the background currents for $\mathrm{K}^{+}$and $\mathrm{Na}^{+}$with the known ultra-rapid $\mathrm{K}^{+}$channel and hyperpolarization activated $\mathrm{K}^{+} / \mathrm{Na}^{+}$ channel currents as shown in Figure 1. This approach for identifying a theoretical model of hiPSC-CM function has three major advantages: 1) relative variations in electrophysiological 
function can be identified between different batches and cell lines of hiPSC-CMs or different lines of hiPSC-CMs derived from different donors; 2) functions of hiPSC-CMs with different maturation status can be described by utilizing expression data at each maturation time point; and 3) this model can be directly compared to hAVCM function where relative expression is set to one for all ion channels, pumps and transporters. In the methods section, we outline how we have modified the ORd model to simulate electrophysiology of hiPSC-CMs yet preserve the ability for the model upon reparameterization to recapitulate the hAVCM phenotype. In the results below, we will use this model to represent the electrophysiology of three batch variants across two different commercial cell lines where changes in the action potential duration at $90 \%$ repolarization $\left(\mathrm{APD}_{90}\right)$ are identified to show qualitative agreement with experimental results. Finally, we predict with this expression-based hiPSC-CM model how concurrent blocking of fast and slow components of the $\mathrm{Na}^{+}$channel, rapid delayed rectifier $\mathrm{K}^{+}$channel and L-type $\mathrm{Ca}^{2+}$ channel influences $\mathrm{APD}_{90}$ and proarrhythmia potential.

\section{Results}

\section{Simulating a typical action potential morphology of iPSC-CMs}

To represent the differences in electrophysiological properties between hiPSC-CMs and hAVCMs, the ORd model was modified to simulate electrophysiology of hiPSC-CMs by scaling conductances and permeabilities with respect to relative gene expressions of the ion channels, exchangers, pumps and receptors (Table 1). It is assumed that the relative gene expression between hAVCMs and hiPSC-CMs of the gene encoding a given channel, exchanger, etc. can be used to linearly scale its associated conductance or permeability in the hiPSC-CM model. Simulations were performed for each of the 3 different batches from two hiPSC-CM lines produced from a commercial supplier of hiPSC-CMs ${ }^{7}$ along with the expression in hAVCM which we assumed to be represented by the ORd model simulated at its published parameters. A simulated AP profile of the hiPSC-CM resembles those obtained experimentally using patch clamp techniques $^{13}$ and membrane potential dyes reflecting APs published previously ${ }^{15}$. Our simulations also indicate that pacing frequency can significantly change morphologies of AP profiles and shows a predicted shorter $\mathrm{APD}_{90}$ for Cor4U cell lines than for the iCell lines (Figure $2 \mathbf{a}, \mathbf{b}$ ).

\section{Elevated resting membrane potential in hiPSC-CM}

As has been previously recorded experimentally ${ }^{13,14}$, hiPSC-CMs have a higher resting membrane potential than that of hAVCMs. Our expression-based hiPSC-CM model, parameterized to represent iCell1, simulated an elevated resting membrane potential of hiPSC-CM to be $-81.0 \mathrm{mV}$ at $1 \mathrm{~Hz}$ and $-71.5 \mathrm{mV}$ at $0.5 \mathrm{~Hz}$, which are both significantly higher than that $(-87.9 \mathrm{mV})$ of hAVCM (Figure 3). Because of the elevated resting membrane potential in hiPSC-CMs the inactivation gate of fast $\mathrm{Na}^{+}$channels do not entirely reset. Therefore, it is posited that the fast $\mathrm{Na}^{+}$ channel current, $I_{N a}$, does not contribute significantly to the initiation of the hiPSC-CM excitation. Instead an influx of calcium ions through L-type Ca channel, $I_{C a L}$, initiates the excitation. Comparison between the simulated $I_{N a}$ (Figure $3 \mathbf{c}, \mathbf{d}$ ) and $I_{C a L}$ (Figure $3 \mathbf{e}, \mathbf{f}$ ) of hAVCM and 
hiPSC-CM shows an increased $I_{C a L}$ and reduced $I_{N a}$ contribution in hiPSC-CMs which were exacerbated further by reducing pacing frequencies to $0.5 \mathrm{~Hz}$. The maximal rate of AP depolarization $\left(\mathrm{dV} / \mathrm{dt}^{\max }\right.$ ), of hAVCM and hiPSC-CMs are $342 \mathrm{mV} / \mathrm{ms}$ and $134 \mathrm{mV} / \mathrm{ms}$ respectively, indicating the hiPSC-CM's reduced maximum rate of membrane potential depolarization with reduced $I_{N a}$ contribution. These $\mathrm{dV} / \mathrm{dt}^{\max }$ values are calculated by finding the steepest section of the AP upstroke when the time from AP initiation to peak voltage is divided into 100 sections (see Supplemental Table 1). These simulation $\mathrm{dV} / \mathrm{dt}^{\max }$ values are larger than those reported experimentally ${ }^{16}$ likely because the experimental measures are sampled at a lower frequency. However, the same relative observation can be seen with the $\mathrm{dV} / \mathrm{dt}^{\text {max }}$ of hAVCMs are larger than that of hiPSCCMs.

\section{$I_{C a L}$ and $I_{N a}$ contributions to electrophysiology of hiPSC-CM and hAVCM}

To analyze the extent to which $I_{C a L}$ and $I_{N a}$ current influences the shape of AP and calcium transient (CaT) profiles in hiPSC-CMs, we modulated the L-type $\mathrm{Ca}^{2+}$ or fast $\mathrm{Na}^{+}$channel conductance in each model with the ORd model representing an hAVCM and the iCell1 as the representative hiPSC-CM (Figure 4). The L-type $\mathrm{Ca}^{2+}$ or fast $\mathrm{Na}^{+}$channel conductances were varied in each model from 0 to 5 times that of hAVCM while the remaining ion channels, exchangers, etc. were held constant at the hAVCM and hiPSC-CM values. Without $I_{C a L}$ current, hiPSC-CM and hAVCM did not exhibit a plateau phase (Figure $4 \mathbf{a}, \mathbf{b}$ ). While increasing $I_{C a L}$ conductance elongated AP duration in hAVCMs and hiPSC-CMs, even a small increment (e.g., $g_{C a L} / g_{C a L, 0}=0.5$ ) induced the plateau phase of hiPSC-CM. An increasing $\mathrm{Ca}^{2+}$-entry via the $I_{C a L}$ channel above the level of hAVCM $\left(g_{C a L} / g_{C a L, O}>1\right)$ changed peak of CaT more significantly in hAVCMs than in hiPSC-CMs (Figure 4c, d).

Changing the fast $\mathrm{Na}^{+}$channel conductance had little effects on AP profiles in the hAVCM (Figure $4 \mathbf{e}$ ) but changed amplitudes of CaTs drastically (Figure 4g). Even without the $I_{N a}$ current $\left(g_{N a} / g_{N a, 0}=0\right)$, the pacing current $(-80 \mathrm{mV}$ pulse for $1 \mathrm{~ms})$ in the simulation was enough to activate $I_{C a L}$ and generate an AP in the hAVCM. An increasing $I_{N a}$ current elevated the maximal slope of the membrane potential, $(\mathrm{dV} / \mathrm{dt})^{\max }$ in the hAVCM from $80 \mathrm{mV} / \mathrm{s}$ at $g_{N a} / g_{N a, 0}=0$ to 435 $\mathrm{mV} / \mathrm{s}$ at $g_{\mathrm{Na}} / g_{\mathrm{Na}, 0}=5$ (sampled at 100 time divisions on upstroke). Conversely, the changes in $I_{\mathrm{Na}}$ current had almost no effect on the hiPSC-CM profiles of AP and a very little effect on CaT (Figure 4g, h).

\section{Modulating $I_{K I}$ but not $I_{h}$ restores resting membrane potential}

To explore roles of $I_{K I}$ and hyperpolarization-activated $\mathrm{Na}^{+} / \mathrm{K}^{+}$current (also known as the funny current), $I_{h}$, in the elevated resting membrane potential of hiPSC-CM (again iCell1), we modulated $I_{K l}$ currents in the hiPSC-CM model. While keeping $I_{h}$ constant, increasing $I_{K I}$ current from zero or a low level indicated by expression data in hiPSC-CMs to that of an hAVCM lowered the elevated resting membrane potential back to $\sim-88 \mathrm{mV}$ (Figure 5a). Changing the conductance of $I_{h}$ on the other hand, had little effect on changing the AP regardless of the $I_{K 1}$ expression levels $\left(g_{K I} / g_{K 1,0}=1\right.$ or 0 , Figure $\left.5 \mathbf{b}, \mathbf{c}\right)$. After restoring the resting membrane potential by elevating $I_{K l}$, 
the simulation shows that $I_{N a}$ becomes a more active player in depolarizing the hiPSC-CM's membrane potential.

\section{Reduced contribution of sarcoplasmic reticulum (SR) in $\mathrm{Ca}^{2+}$ transients of hiPSC-CMs}

To see the functional difference in calcium induced calcium release from the SR in hiPSC-CMs, we varied the flux through the ryanodine receptor (RyR) and sarcoplasmic/endoplasmic reticulum $\mathrm{Ca}^{2+}$ ATPase (SERCA) in both models. Both independent and concurrent changes in flux of RyR and SERCA had little effect on AP profiles (Figure $6 \mathbf{a}, \mathbf{b}, \mathbf{c}$ ). CaT showed little change when only RyR flux was varied (Figure 6d) and moderate change when only SERCA flux was varied (Figure 6e). Combined effects of changing RyR and SERCA had large effects on CaT (Figure 6f). In the hiPSC-CM simulation of Figure $4 \mathbf{d}$ at a $g_{C a L} / g_{C a L, 0}=5$ we see a maximum amplitude of $\sim 0.35 \mu \mathrm{M}$ which is similar to the maximal amplitudes in Figure $6 \mathbf{d}$ however bringing SERCA flux up to hAVCM levels independently (Figure 6e) or concurrently with RyR flux (Figure 6f) results in cytosolic $\mathrm{Ca}^{2+}$ concentrations of about 0.5 and $1.25 \mu \mathrm{M}$ respectively which is much higher than levels predicted in the hAVCM by the ORd model (Figure $4 \mathbf{c}$ at $g_{C a L} / g_{C a L, 0}=1$ ).

\section{hAVCM and hiPSC-CM proarrhythmic risk assessment of cardiac drugs}

To visualize the differences in proarrhythmic sensitivities between hAVCM and hiPSC-CM we theoretically blocked the rapid delayed rectifier $\mathrm{K}^{+}$, fast $\mathrm{Na}^{+}$(including the slow $\mathrm{Na}^{+}$component) and L-type $\mathrm{Ca}^{2+}$ channels from 0 to $100 \%$ in different combinations (Figure 7). Blocking of each channel was accomplished by scaling the conductance of that channel between 0 to 1 ( 1 being normal conductance and 0 being a complete block). Each set of blocking parameters defined a position in the $\mathrm{K}^{+}, \mathrm{Na}^{+}$and $\mathrm{Ca}^{2+}$ space and the $\mathrm{APD}_{90}$ of the resulting AP simulation was calculate at each position. $\mathrm{APD}_{90}$ values were scaled across a blue to red spectrum and we added separate colors for: arrhythmia including EAD (purple) and unstable AP or no depolarization (black). The 3D plots revealed that hiPSC-CM are more susceptible to $I_{K r}$ and $I_{C a L}$ block and exhibit larger regions in the 3D space of arrhythmia as indicated in purple and black at both $1 \mathrm{~Hz}$ (Figure $7 \mathbf{a}, \mathbf{b}$ ) and at $0.5 \mathrm{~Hz}$ (Figure $7 \mathrm{c}, \mathrm{d}$ ). The interplay between $I_{K r}$ and $I_{C a L}$ block describes much of the effects seen as is observed when we project the entire $3 \mathrm{D}$ space onto a $\mathrm{K}^{+}$and $\mathrm{Ca}^{2+}$ plane (Figure 7e-h). 2D cross-sectional plots of $I_{N a}$ and $I_{C a L}$ block keeping $I_{K r}$ constant shows no effect on $\mathrm{APD}_{90}$ as a function of $I_{N a}$ block (Supplement Figure $\mathrm{S} 1$ ).

Analyzing projections of 3D plots onto a 2D plane defined by $I_{K r}$ and $I_{C a L}$ axis (Figure 7e-h) further clarified that an $I_{C a L}$ inhibition can mitigate $\mathrm{APD}_{90}$ elongation and/or arrhythmic AP profiles induced by $I_{K r}$ blocking especially in hiPSC-CMs. The simulations also show that reducing the pacing frequencies enlarged arrhythmia sensitivity zone (purple and black) in both hAVCM and hiPSC-CM. At the lower pacing frequency (Figure $6 \mathbf{g}, \mathbf{h}$ ), an increased $I_{K r}$ block moves AP profiles from moderate $\mathrm{APD}_{90}$ elongations rapidly into regions of arrhythmia sensitivity zone skipping over extremely elongated $\mathrm{APD}_{90}$ zones indicated by red and orange. 


\section{Discussion}

In this study, previous measurements of relative gene expression of key ion channels, exchangers, pumps and receptors between hAVCM and hiPSC-CM cell lines ${ }^{7}$ has been used to modify an existing mathematical model of the hAVCM (ORd model $\left.{ }^{14}\right)$ resulting in a novel hiPSC-CM electrophysiology and ion handling model. By using relative expression data which can be obtained across different cell lines and even batches of a given cell line we are able to develop a theoretical hiPSC-CM model that qualitatively represents the variation in cell function and can be matched to a specific experiment preparation. This expression-based hiPSC-CM model not only exhibited previously observed characteristic differences between hiPSC-CMs and hAVCMs, but qualitatively reproduced the trends in $\mathrm{APD}_{90}$ observed across the 3 different batches of 2 different cell lines by Huo et al. ${ }^{7}$ (Table 2). The experimentally observed characteristic differences reported from various laboratories have been summarized previously by Hoekstra et al. ${ }^{16}$ and include 1) an elevated resting membrane potential in an hiPSC-CM (-57 to -75mV) when compared to an hAVCM $(-81.8$ to $-87 \mathrm{mV}), 2)$ a reduced maximal upstroke velocity, $(\mathrm{dV} / \mathrm{dt})_{\max }$, in hiPSC-CMs, 3) a longer $\mathrm{APD}_{90}$ in hiPSC-CM than hAVCM and 4) spontaneously beating of hiPSC-CMs. Our expression based hiPSC-CM model exhibits the first three observed characteristics except in the case of modeling the Cor4U lines where both model and observation show a shorter $\mathrm{APD}_{90}$ in contradiction with the third observation by Hoekstra et al. ${ }^{16}$ The expression based hiPSC-CM model does not exhibit spontaneous beating, also known as automaticity, however this is likely because we have removed the ohmic-like $\mathrm{Na}^{+}$background leak current in the model which are typically used to drive resting membrane depolarization and spontaneous beating frequency.

This new expression based hiPSC-CM model has distinct advantages over the most recent hiPSC-CM model by Paci et al. ${ }^{12}$ which is based on patch clamp data from Ma et al. ${ }^{13}$. First and foremost, this model can be developed from expression data alone and does not rely on expensive and time-consuming patch clamp data for each critical ion channel driving hiPSC-CM electrophysiology. Expression data can be tractably obtained from multiple cell lines and therefore can reflect variation in hiPSC-CM function across different cell lines or at different maturation time points. This opens the door to parameterizing hiPSC-CM models which capture the variability needed to reflect patient specific function. Another point of note is that we have eliminated the background leak currents of $\mathrm{Na}^{+}$and $\mathrm{K}^{+}$currents in the model and replaced them with the ultra-rapid $\mathrm{K}^{+}$and hyperpolarization activated $\mathrm{Na}^{+} / \mathrm{K}^{+}$channel currents $\left(I_{K u r}\right.$ and $\left.I_{h}\right)$. The $I_{K u r}$ and $I_{h}$ currents can be quantified through relative expression and therefore this hiPSC-CM model does not rely on unidentifiable leak currents to drive resting membrane depolarization and spontaneous beating. The Paci et al. model ${ }^{12}$ relied on a large $\mathrm{Ca}^{2+}$ leak current to drive resting membrane potential depolarization to represent automaticity which is not used here.

This hiPSC-CM model showed a slowly depolarizing diastolic membrane potential resulting in an elevated resting membrane potential that was especially evident at slow pacing frequencies. A higher resting membrane potential reduces the functional availability of fast $\mathrm{Na}^{+}$channels since 
the inactivation gate cannot fully reset and the reduction in $\mathrm{Na}^{+}$influx is further reduced by the lower current density of the fast $\mathrm{Na}^{+}$channel as indicated by reduced SCN5A expression across all batches of the two hiPSC-CM lines. Shifting the major contributor of CM depolarization from fast $I_{N a}$ to relatively slower $I_{C a L}$ explains the lowered dV/dt ${ }^{\max }$ in hiPSC-CMs. Analysis of the simulations shown in Figure 5 identified that a significantly reduced expression of $I_{K I}$ is the primary cause of slowly elevating resting membrane potential as demonstrated experimentally by reintroducing the $I_{K I}$ channel through forced expression in human embryonic stem cell-derived cardiomyocytes $^{11}$ and electrophysiologically in hiPSC-CMs ${ }^{11,17}$.

This expression based hiPSC-CM model is also able to quantitatively capture the relatively immature sarcoplasmic reticulum (SR) phenotype known to exist in hiPSC-CMs ${ }^{18}$. Previously reported electron micrographic observations of the immature SR showing a lack of T-tubules in hiPSC-CMs ${ }^{19}$ that belies the significantly reduced expression of RyR and SERCA. This immature phenotype is believed to play a role in the longer $\mathrm{APD}_{90}$ typically observed in hiPSC-CMs but longer $\mathrm{APD}_{90}$ also involves the interplay between the increased L-type $\mathrm{Ca}^{2+}$ channel and relatively unchanged rapid delayed rectifier $\mathrm{K}^{+}$channel currents as reflected in the expression data (Table 1). Increased $I_{C a L}$ conductance results in a higher maximum L-type $\mathrm{Ca}^{2+}$ currents in hiPSC-CMs; however, the reduced release and uptake of $\mathrm{Ca}^{2+}$ through the RyR and SERCA pump, respectively, leaves in question the ultimate profile of the calcium transient $(\mathrm{CaT})$ for each cycle. The simulations for a normal hAVCM shown in Figure $4 \mathbf{c}$ at $g_{C a L} / g_{C a L, 0}=1$ and the iCell 1 shown in Figure $4 \mathbf{d}$ at $g_{C a L} / g_{C a L, 0}=5$ indicate that the peak cytosolic $\mathrm{Ca}^{2+}$ for the hiPSC-CM is actually higher and decays more slowly than in the hAVCM. However, reduced RyR in hiPSCCMs results in a sensitivity of cytosolic $\mathrm{Ca}^{2+}$ to changes in $g_{\text {CaL }}$ that was smaller than in hAVCMs. While the SR in hAVCMs efficiently removed an elevated intracellular $\mathrm{Ca}^{2+}$ in diastole, the capacity of hiPSC-CMs to remove intracellular $\mathrm{Ca}^{2+}$ during diastole was compromised. This is due to the reduced ability of the SR in hiPSC-CMs to take up intracellular $\mathrm{Ca}^{2+}$ through SERCA.

Calcium ion is known to be the mediating signaling molecule responsible for translating action potential into cardiac contraction. The gene expression data (Table 1 and Huo et al. ${ }^{7}$ ) reveals that in hiPSC-CMs both RyR and SERCA pump are expressed at a much lower level when compared to hAVCMs. Therefore, the calcium ions in the cytosol enter mainly through $I_{C a L}$ which cannot produce the rapid change in $\mathrm{Ca}^{2+}$ usually observed in hAVCMs. This immature SR phenotype is similar to that observed in CMs during cardiac development ${ }^{18}$.

Simulations of hiPSC-CM (iCell1) over a range of fast $\mathrm{Na}^{+}$channel conductances (Figure 4h) and inward rectifier $\mathrm{K}^{+}$conductance (implied by Figure $5 \mathbf{e}$ ) also show little sensitivity on cytosolic $\mathrm{Ca}^{2+}$. Independently increasing the RyR in the hiPSC-CM to baseline hAVCM levels makes little difference in the $\mathrm{CaT}$ and independently increasing the SERCA flux shows a small change in CaT. However, increasing both concurrently in the hiPSC-CM makes a dramatic difference in 
$\mathrm{CaT}$. Since the CaT will be important to predict accurately as we measure and analyze force generation in hiPSC-CMs, understanding how each ion channel, pump and receptor involved in the composition of the CaT will be important.

While we do see a depolarized resting membrane potential in our expression-based hiPSC-CM model, the membrane potential does not rise to the threshold $(-40 \mathrm{mV})$ needed to spontaneously fire. There are two explanations for this lack of automaticity in the model. First, the combination of the absence of the $\mathrm{Na}^{+}$and $\mathrm{K}^{+}$background leak currents, which have been eliminated in this model, and the inclusion of $I_{h}$, which is the major depolarizing current used to replace the omitted background currents are not able to generate a constant depolarization as the membrane potential drifts away from the typical resting membrane potential of $\sim-88 \mathrm{mV}$. Automaticity could be achieved by inserting a small background $\mathrm{Na}^{+}$or changing the conductance of the retained background $\mathrm{Ca}^{2+}$, however full AP time courses would be necessary to identify the background channel conductance needed to achieve automaticity. The second reason is that automaticity may be driven by a single or small group of cells that have enough depolarization to generate an AP. AP generation from this region then would drive depolarization in neighboring hiPSC-CMs propagating across the entire tissue. Observations of CaT propagation in a two-dimensional space $\left(8 \times 8 \mathrm{~mm}^{2}\right)$ made in our lab (result not shown) show that CaT was initiated from a single site and rarely from more than that one site. The pacemaker-like hiPSC-CMs may also express T-type Ca channel that can be depolarized at lower membrane potential and drive automaticity but would not show up as significantly present in our expression data.

The strength of this theoretical approach is that we are now able to visualize the proarrhythmia potential of drugs that block the typical cardiomyocyte ion channel targets such as fast $\mathrm{Na}^{+}, \mathrm{L}-$ type $\mathrm{Ca}^{2+}$ and rapid delayed rectifier $\mathrm{K}^{+}$channels in both a normal hAVCM and a representative hiPSC-CM. Figure 7, where the hiPSC-CM is represented by iCell1, shows: 1) hiPSC-CMs are predicted to have a higher proarrhythmia risk in response to ion channel blockage than hAVCMs 2) proarrhythmia risk associated with $I_{K r}$ blockage can be mitigated by concurrent blocking of $I_{C a L}$ and 3) blocking of fast $\mathrm{Na}^{+}$channels has little effect on proarrhythmia risk (also see Supplement Figure S1). In addition, we are able with this methodology to understand why different hiPSC-CM cell lines might actually show different responses. iCell lines would be posited here to exhibit a higher proarrhythmia risk than Cor4U cell lines since the iCell lines exhibit a longer $\mathrm{APD}_{90}$ than Cor4U even before a theoretical blocking agent is applied.

\section{Limitations}

In this study, we are using relative expression data of each hiPSC-CM cell line and batch with respect to expression levels to hAVCM. We are assuming that the hAVCM expression levels represent the conductances, permeabilities and maximal fluxes given in the ORd model. This assumption does not affect the qualitative observations we are discussing in this study. However, to match AP and CaT time courses along with generating a model that spontaneously beats at the 
correct frequency, the reference hAVCM model must be identified with a series of experiments similar to that proposed by Krogh-Madsen et al. ${ }^{20}$.

The $\mathrm{Ca}^{2+}$ release kinetics through RyR in the original ORd model depends on the conductance of $I_{C a L}$, SR junctional space $\mathrm{Ca}^{2+}$ concentration, and activity of $\mathrm{CaMKII}^{21}$. This description is representative of SR function in hAVCMs where the dyadic space formed between T-tubule and sarcoplasmic reticulum (SR) and the pairing of RyR and L-type calcium channel is generally accepted. This structural description, however, may not be present in hiPSC-CMs that have significantly reduced RyR and SERCA expression and are thought to be not as tightly coupled to the release of $\mathrm{Ca}^{2+}$ through L-type $\mathrm{Ca}^{2+}$ channels in the dyadic space. As more detail becomes known about the structural and functional details of the dyadic space and SR function in hiPSC-CMs, we would be able to modify SR function based on more than just RyR and SERCA expression.

\section{Conclusion}

We have modified a widely accepted model for the electrophysiology and ion handling in an $\mathrm{hAVCM}^{14}$ using relative expression data of ion channel, exchange, pump and receptor protein encoded genes to develop a novel model of the hiPSC-CM. This model can represent variability in hiPSC-CM function across different cell lines and cell line batches to qualitatively represent difference in action potential duration. The hAVCM model used in this development is currently supported by CiPA for the testing of proarrhythmia risk ${ }^{22}$. This expression-based hiPSC-CM model suggests that using hiPSC-CMs for testing of proarrhythmia risk of drug compounds over predicts the risk for $\mathrm{K}^{+}$and $\mathrm{Ca}^{2+}$ ion channel blocking compounds.

\section{Methods}

\section{Theoretical model of human induced stem cell derived cardiomyocytes}

This study modified the ORd hAVCM model ${ }^{14}$ to reflect the relative differences in the expression of eight different transmembrane ion channels, the $\mathrm{Na}^{+} / \mathrm{Ca}^{+}$exchanger along with ryanodine receptor and the calcium pump in the SR in hiPSC-CMs against those in hAVCMs ${ }^{7}$. The ORd model is a comprehensive electrophysiology and ion handling model that was developed based on experimental measurements on dynamic properties of ion channels and pumps using isolated healthy hAVCMs. The relative expression data is then used to scale the conductance of, permeability of, or flux through each specific channel, exchanger, pump or receptor in the theoretical model creating an expression-based hiPSC-CM model (see example set of equations in the Supplement). It is assumed that there is no change in the gating or binding kinetics in any of the selected transport mechanisms and the number of fully functional channels and transporters in cells is a liner function of gene expression.

The gene expression analysis of hiPSC-CMs ${ }^{7}$ detected ultra-rapid $\mathrm{K}^{+}$(Kv1.5, KCNA5) channels and hyperpolarization-activated cation $(\mathrm{HCN} 2$ and $\mathrm{HCN} 4)$ that are not represented in the ORd 
model. The Kv1.5 channel protein has been detected at equal levels in human atrial and ventricular cardiomyocytes ${ }^{23}$ however the ultra-rapid $\mathrm{K}^{+}$current $\left(I_{K u r}\right)$ is either non-existent or reduced in hAVCMs ${ }^{24}$. Similarly, the gene expression of HCN2 and HCN4 is seen in both atrial (specifically the sinoatrial node (SAN) and ventricular tissue, however the ratio of HCN4/HCN2 is much larger in the SAN than in the ventricle. In addition, the total amount of HCN has been reported to be as much as 7.5 times lower in ventricular cardiomyocytes than in SAN cells, suggesting a reduced current density in hAVCMs.

To represent these two missing channel currents in the ORd model we looked at previous modeling studies of atrial and ventricular myocyte electrophysiology. The ultra-rapid delayed rectifier $\mathrm{K}^{+}$current was formulated based on the human atrial cell electrophysiology model by Cortmanche et al. ${ }^{25}$ with the maximal conductance reduced about 6 -fold from values found in human atrial cells as indicated experimentally ${ }^{24}$. For the hyperpolarization-activated cation current, $I_{h}$, we used the model from Kurata et al. ${ }^{26}$ for the HCN2 channel based on data from Moroni et al. $^{27}$. The advantage of using the Kurata et al. model is that instead of lumping the inflow current from $\mathrm{Na}^{+}$and outflow current from $\mathrm{K}^{+}$into a net current, each ionic current is represented separately. While both HCN2 and HCN4 are present in ventricular cardiomyocytes it has been shown in mouse ventricular tissue that over $75 \%$ of the $\mathrm{HCN}$ expression is that of the HCN2 isoform ${ }^{28}$. However, expression data from Huo et al. ${ }^{7}$ shows the opposite trend in hAVCM cells with $75 \%$ of the HCN expression in the form of HCN4.

The major functional difference between the four different isoforms in the HCN family is their rate of activation with $\mathrm{HCN} 1$ having the most rapid and $\mathrm{HCN} 4$ having the slowest gating kinet$\mathrm{ics}^{29}$. Even though there is some faster gating HCN2 in ventricular cardiomyocytes, measurements of the gating time constant, $\tau_{h}$, in human embryonic stem cell derived cardiomyocytes (heSCMs) is on the order of $2000 \mathrm{~ms}$ in the range of -110 to $-90 \mathrm{mV}^{30}$. This time constant is closer to the gating kinetics of the $\mathrm{HCN} 4$ isoform so we replaced the Kurata et al. gating kinetics with that used in the Paci et al. hiPSC-CM model ${ }^{12}$. Because these two ion-channel currents $\left(I_{K u r}\right.$ and $I_{h}$ ) were not represented in the ORd model instead of adding them as additional currents we used them to replace the background $\mathrm{Na}^{+}$and $\mathrm{K}^{+}$channels in the original ORd model. The $\mathrm{Ca}^{2+}$ background channel current was retained. The maximal conductances of these two channels in the hAVCM led to currents that were close to the ORd background $\mathrm{Na}^{+}$and $\mathrm{K}^{+}$currents in hAVCMs and when changed as a ratio of expression to represent the hiPSC-CM cell lines resulted in an AP phenotype in diastole like that observed experimentally. Replacement of the two background channels with ion channels with conductance fixed to the expression data limited our ability to generate automaticity by changing conductance; however, a depolarized resting membrane potential was achieved with the expression-based modification of model conductances alone.

The dyad space was retained in the ORd model even though there is evidence that the formation of t-tubules is reduced in hiPSC-CMs. This will not necessarily affect the shape of the action po- 
tentials in the models used here but must be considered when sarcomere force generation is simulated from the cytosolic calcium transients. A representation of the modified ORd/hiPSC-CM model and the modifications made are shown in Figure 1.

An important feature of the ORd model is that given appropriate initial conditions the model goes to a steady state with stable APs as well as cytosolic concentrations of $\mathrm{Na}^{+}, \mathrm{Ca}^{2+}$ and $\mathrm{K}^{+}$ $\left(\left[\mathrm{Na}^{+}\right]_{i},\left[\mathrm{Ca}^{2+}\right]_{\mathrm{i}}\right.$ and $\left[\mathrm{K}^{+}\right]_{\mathrm{i}}$, respectively). To check that this remained the case with the new inserted $I_{K u r}$ and $I_{h}$ for the hAVCM and all six hiPSC-CM parameterized model simulations we calculated the change at 10 different time points of $\mathrm{V}_{\mathrm{m}},\left[\mathrm{Na}^{+}\right]_{\mathrm{i}},\left[\mathrm{Ca}^{2+}\right]_{\mathrm{i}}$ and $\left[\mathrm{K}^{+}\right]_{\mathrm{i}}$ between two successive APs. A steady state was reached in all cases with the error in each of the four variables dropping below a $0.1 \%$ change.

Simulations were performed for three batches from two different hiPSC-CM lines along with the hAVCM at stimulated at frequencies of 1 and $0.5 \mathrm{~Hz}$. The stimulation was in the form of a step function of an applied voltage with magnitude $-80 \mathrm{mV}$ for $1 \mathrm{~ms}$. From these six different representations of an hiPSC-CM, we chose to look at a single representative hiPSC-CM (iCell1) and compare it to the simulated response of the hAVCM. All simulations reached a steady state according to the criteria previously mentioned. All simulations were coded and run in Matlab (Matlab, Inc., Natick, MA, USA).

\section{Expression data and experimental methods}

Expression data was obtained from researchers at the FDA and methods to obtain expression for the 3 batches of 2 different cell lines are described in detail in the study by Huo et al. ${ }^{7}$. Briefly, three batches of hiPSC-CMs from two vendors and one hAVCM line were prepared according to the vendors specifications. The iCell cells were obtained from Cellular Dynamics International (Madison, Wisconsin), the Cor4U cells were obtained from Axiogenesis (Nattermannalee, Germany) and the hAVCMs were obtained from Biochain (San Francisco, California). Total RNAs were isolated at day 15 for the iCells and day 9 for the Cor4U cells and real-time qRT-PCR was performed on the cells to obtain expression of 16 cardiac genes related to the ion channels, exchangers, pumps and receptors used to quantify conductances and fluxes used here. The qRTPCR expression values for the hAVCM were used to normalize the hiPSC-CM expression data. In addition, observations from the study of Huo et $\mathrm{al}^{7}$ of field potential duration (FPD) were compared to our simulations results for $\mathrm{APD}_{90}$ to confirm trends.

\section{Disclaimer}

The views presented in this article do not necessarily reflect those of the Food and Drug Administration. The authors declare that there are no conflicts of interest regarding the publication of this article. 


\section{Table Legends}

Table 1. Relative gene expression data for eleven ion channels, pumps, exchangers and receptors in hAVCM and 3 batches from two hiPSC-CM cell lines (iCell and Cor4U). These expression data are from Figure 1 of the study by Huo et al. ${ }^{7}$ where hAVCM is represented by hALV.

Table 2. Beating rate (BR) and field potential duration (FPD) for iCell and Cor4U hiPSC-CM lines from Huo et al. . 
Table 1.

\begin{tabular}{|c|c|c|c|c|c|c|c|c|c|}
\hline Gene & $\begin{array}{c}\text { Ion channel, } \\
\text { pump or re- } \\
\text { ceptor }\end{array}$ & $\begin{array}{l}\text { Current } \\
\text { or Flux }\end{array}$ & hAVCM & iCell1 & iCell2 & iCell3 & Cor.4U1 & Cor.4U2 & Cor.4U3 \\
\hline SCN5A & Fast $\mathrm{Na}^{+}$ & $I_{N a}$ & 1.000 & 0.656 & 0.765 & 0.777 & 0.371 & 0.391 & 0.303 \\
\hline CACNA1C & L-Type $\mathrm{Ca}^{2+}$ & $I_{C a L}$ & 1.000 & 4.716 & 4.110 & 5.397 & 3.282 & 2.735 & 1.256 \\
\hline $\mathrm{KCNH} 2$ & $\begin{array}{c}\text { Rapid Delayed } \\
\text { Rectifier } \mathrm{K}^{+}\end{array}$ & $I_{K r}$ & 1.000 & 1.060 & 1.001 & 1.161 & 1.352 & 1.290 & 1.130 \\
\hline KCNQ1 & $\begin{array}{c}\text { Slow Delayed } \\
\text { Rectifier K }\end{array}$ & $I_{K s}$ & 1.000 & 0.987 & 0.976 & 0.965 & 1.100 & 0.966 & 0.892 \\
\hline KCND2 & $\begin{array}{l}\text { Transient Out- } \\
\text { ward } \mathrm{K}^{+}\end{array}$ & $I_{t o}$ & 1.000 & 1.464 & 1.902 & 1.660 & 4.893 & 3.096 & 1.267 \\
\hline KCNA5 & $\begin{array}{c}\text { Ultra-rapid De- } \\
\text { layed Rectifier } \\
\mathrm{K}^{+}\end{array}$ & $I_{K u r}$ & 1.000 & 0.0897 & 0.0913 & 0.0620 & 4.415 & 3.851 & 9.876 \\
\hline HCN4 & $\begin{array}{c}\text { Hyperpolariza- } \\
\text { tion Activated } \\
\mathrm{K}^{+}\end{array}$ & $I_{f}$ & 1.000 & 6.152 & 6.801 & 6.651 & 8.267 & 8.139 & 6.250 \\
\hline $\mathrm{KCNJ} 2$ & $\begin{array}{l}\text { Inward Recti- } \\
\text { fier } \mathrm{K}^{+}\end{array}$ & $I_{K 1}$ & 1.000 & 0.0555 & 0.0544 & 0.0742 & 0.0946 & 0.0633 & 0.0625 \\
\hline SLC8A1 & $\begin{array}{l}\mathrm{Na}^{+} / \mathrm{Ca}^{2+} \mathrm{Ex}- \\
\text { changer }\end{array}$ & $I_{\mathrm{NaCa}}$ & 1.000 & 1.509 & 1.415 & 1.549 & 2.185 & 2.282 & 1.528 \\
\hline RYR2 & $\begin{array}{l}\text { Ryanodine Re- } \\
\text { ceptor }\end{array}$ & $\mathrm{J}_{\text {rel }}$ & 1.000 & 0.0741 & 0.0768 & 0.108 & 0.210 & 0.135 & 0.262 \\
\hline ATP2A2 & $\begin{array}{c}\text { Sarcoplasmic } \\
\text { Reticulum } \mathrm{Ca}^{2+} \\
\text { Pump }\end{array}$ & $\mathrm{J}_{\text {up }}$ & 1.000 & 0.358 & 0.335 & 0.377 & 0.673 & 0.563 & 0.775 \\
\hline
\end{tabular}


bioRxiv preprint doi: https://doi.org/10.1101/236240; this version posted December 18,2017 . The copyright holder for this preprint (which was not certified by peer review) is the author/funder, who has granted bioRxiv a license to display the preprint in perpetuity. It is made available under aCC-BY-NC 4.0 International license.

Table 2.

\begin{tabular}{lcccccc} 
& iCell 1 & iCell 2 & iCell 3 & Cor4U 1 & Cor4U 2 & Cor4U 3 \\
\cline { 2 - 6 } BR (bpm) & $36.1 \pm 1.3$ & $35.8 \pm 1.3$ & $36.7 \pm 1.5$ & $50.9 \pm 7.2$ & $67.3 \pm 4.2$ & $82.2 \pm 3.9$ \\
FPD (ms) & $524.0 \pm 24.7$ & $522.5 \pm 40.5$ & $455.6 \pm 29.0$ & $415.3 \pm 57.4$ & $256.6 \pm 29.8$ & $172.2 \pm 23.9$
\end{tabular}




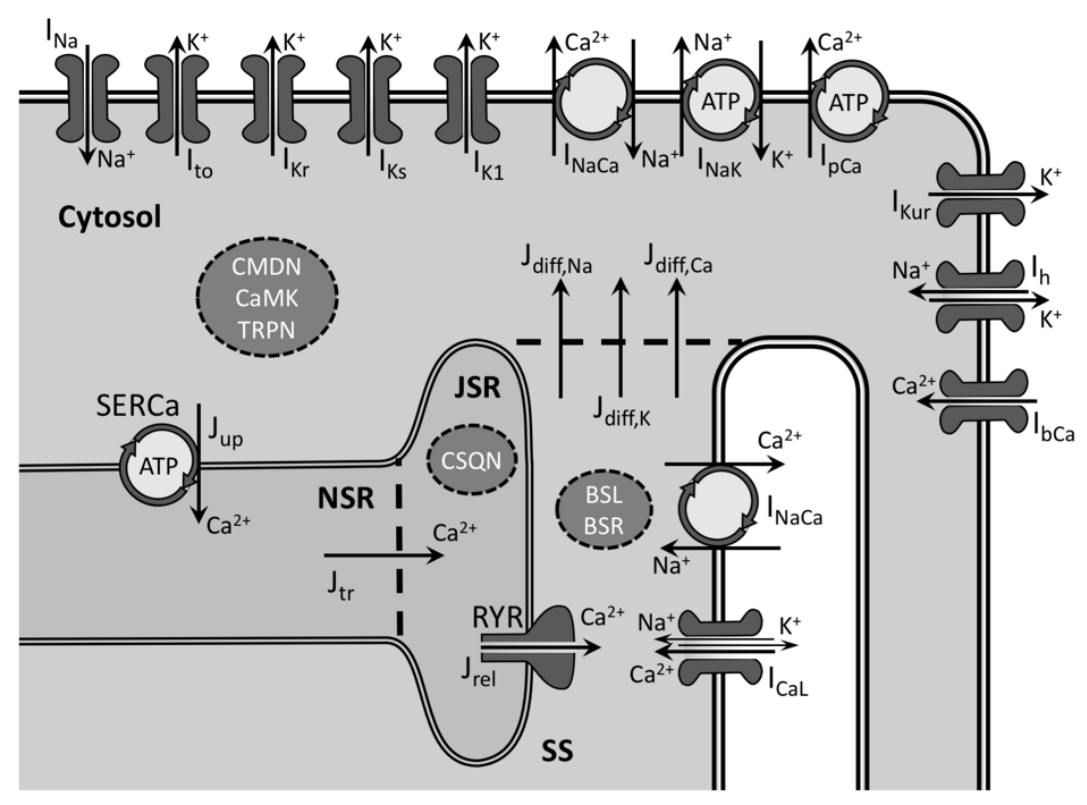

Figure 1. Schematic of the modified ORd model where maximal conductances/permeabilites can be reparameterized to represent the electrophysiology of an hiPSC-CM. Channel, exchanger and pump currents and fluxes are formulated in the same way as in the ORd model: fast $\mathrm{Na}^{+}\left(I_{\mathrm{Na}}\right)$, transient outward $\mathrm{K}^{+}\left(I_{t o}\right)$, rapid delayed rectifier $\mathrm{K}^{+}\left(I_{K r}\right)$, slow delayed rectifier $\mathrm{K}^{+}\left(I_{K s}\right)$, inward rectifier $\mathrm{K}^{+}\left(I_{K I}\right), \mathrm{Na}^{+} / \mathrm{Ca}^{2+}$ exchanger $\left(I_{N a C a}\right), \mathrm{Na}^{+} / \mathrm{K}^{+}$ATPase $\left(I_{N a K}\right)$, plasma membrane $\mathrm{Ca}^{2+}$ pump $\left(I_{p C a}\right)$, L-type $\mathrm{Ca}^{2+}\left(I_{C a L}\right)$, ryanodine receptor $\mathrm{Ca}^{2+}\left(\mathrm{J}_{\text {rel }}\right)$, sarcoplasmic/endoplasmic reticulum $\mathrm{Ca}^{2+}$ pump $\left(\mathrm{J}_{\mathrm{up}}\right)$ and background $\mathrm{Ca}^{2+}\left(\mathrm{I}_{b C a}\right)$. Two background channel currents are omitted - background $\mathrm{Na}^{+}$and $\mathrm{K}^{+}$- and are replaced with an ultrarapid uptake $\mathrm{K}^{+}$current $\left(I_{K u r}\right)$ and a hyperpolarization-activated $\mathrm{Na}^{+} / \mathrm{K}^{+}$current $\left(I_{h}\right)$. Buffering of $\mathrm{Ca}^{2+}$ is implemented in the same manner as in the ORd model: subspace (SS) buffering by the sarcolemmal and sarcoplasmic reticulum membranes (BSL and BSR, respectively), calmodulin, calmodulin kinase and troponin in the cytosol (CMDN, CaMK and TRPN, respectively) and calsequestrin (CSQN) in the junctional sarcoplasmic reticulum (JSR). Note that the total L-type $\mathrm{Ca}^{2+}$ current formulation includes small $\mathrm{Na}^{+}$and $\mathrm{K}^{+}$currents and the total fast $\mathrm{Na}^{+}$current includes both fast and late $\mathrm{Na}^{+}$currents. $\mathrm{Na}^{+} / \mathrm{Ca}^{2+}$ exchangers are distributed across the plasma membrane with $20 \%$ in the t-tubules interacting with the SS and $80 \%$ in the remaining membrane interacting directly with the cytosol. Diffusional fluxes between the SS and cytosol of all three ions are represented by $J_{\text {diff,Na }}, J_{\text {diff }, C a}$ and $\mathrm{J}_{\text {diff,K }}$ and $\mathrm{Ca}^{2+}$ flux between the network sarcoplasmic reticulum (NSR) and JSR by $\mathrm{J}_{\mathrm{tr}}$. 

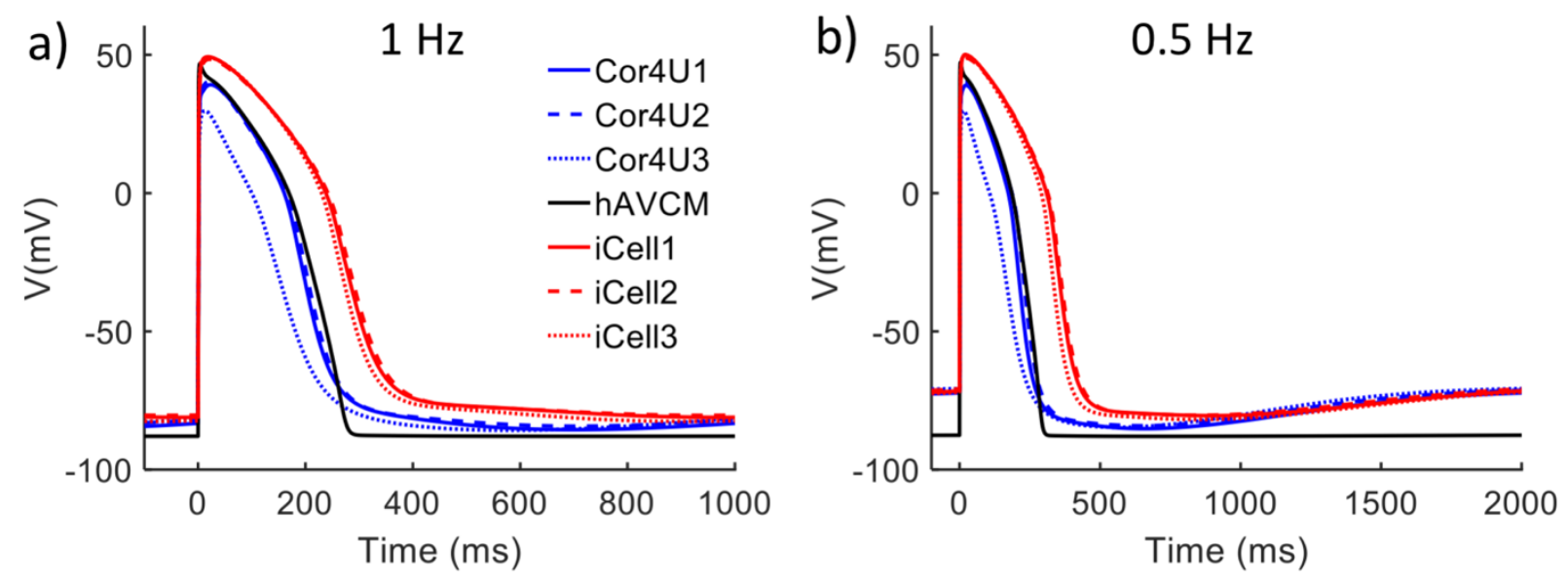

Figure 2. Comparison of simulated action potential profiles generated using gene expression data from two lines of human induced pluripotent stem cell derived cardiomyocytes (hiPSCCMs). Three different batches of hiPSC-CM from each cell line (Cor4U and iCells) were analyzed and compared with an action potential profile of human adult ventricular cardiomyocytes (hAVCMs) at (a) $1 \mathrm{~Hz}$ and (b) $0.5 \mathrm{~Hz}$. 

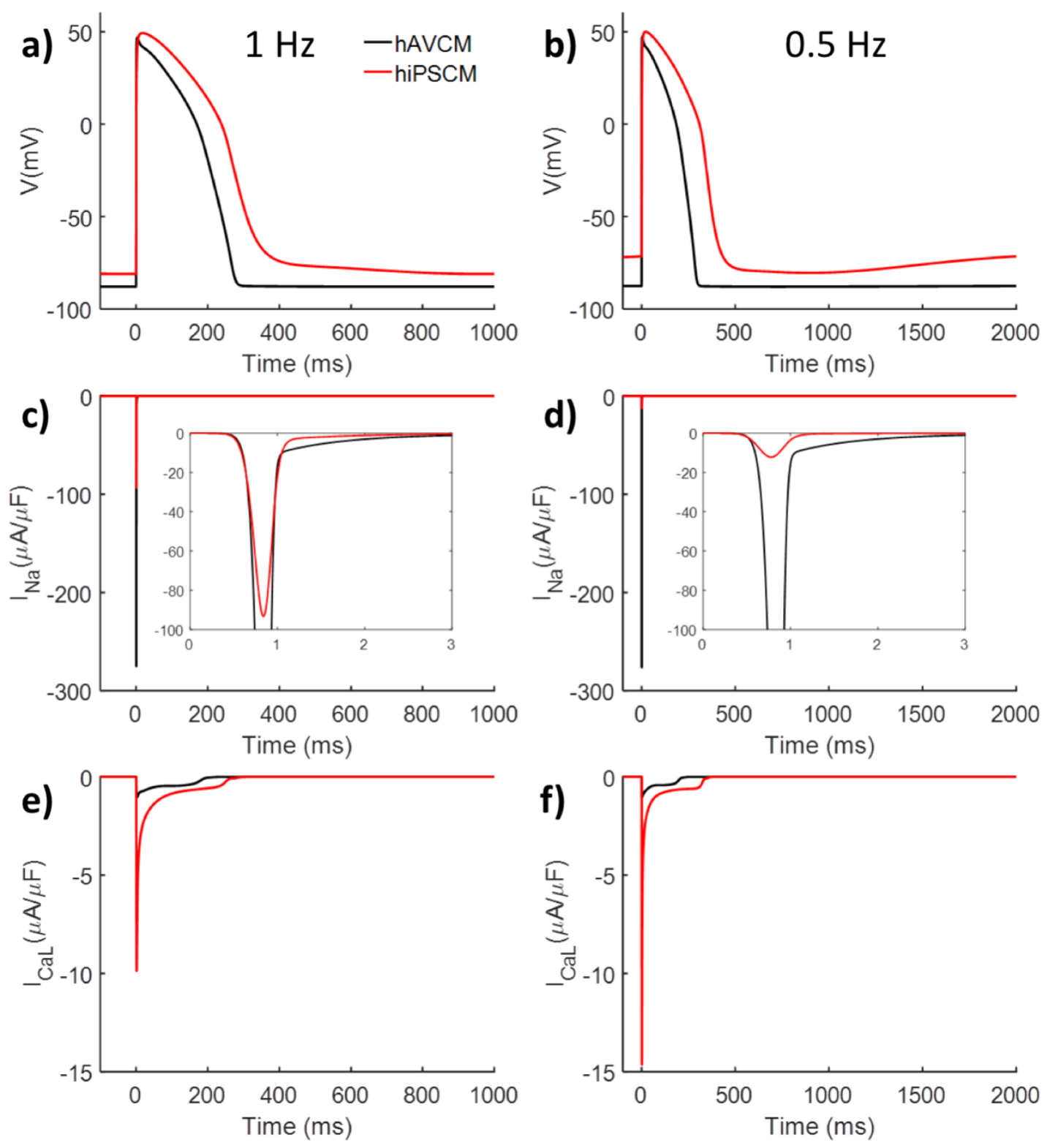

Figure 3. Simulated action potential of hAVCM and hiPSC-CM (iCell1) paced at $1 \mathrm{~Hz}(\mathbf{a})$ and $0.5 \mathrm{~Hz}(\mathbf{b})$. Note that diminished fast $\mathrm{Na}^{+}$current at $1 \mathrm{~Hz}(\mathbf{c})$ and $0.5 \mathrm{~Hz}(\mathbf{d})$ and enlarged L-type $\mathrm{Ca}^{2+}$ current at $1 \mathrm{~Hz}(\mathbf{e})$ and $0.5 \mathrm{~Hz}$ (f) in hiPSC-CM as compared to hAVCM are shown with the largest discrepancy between hiPSC-CM and hAVCM at $0.5 \mathrm{~Hz}$ (e and f). 

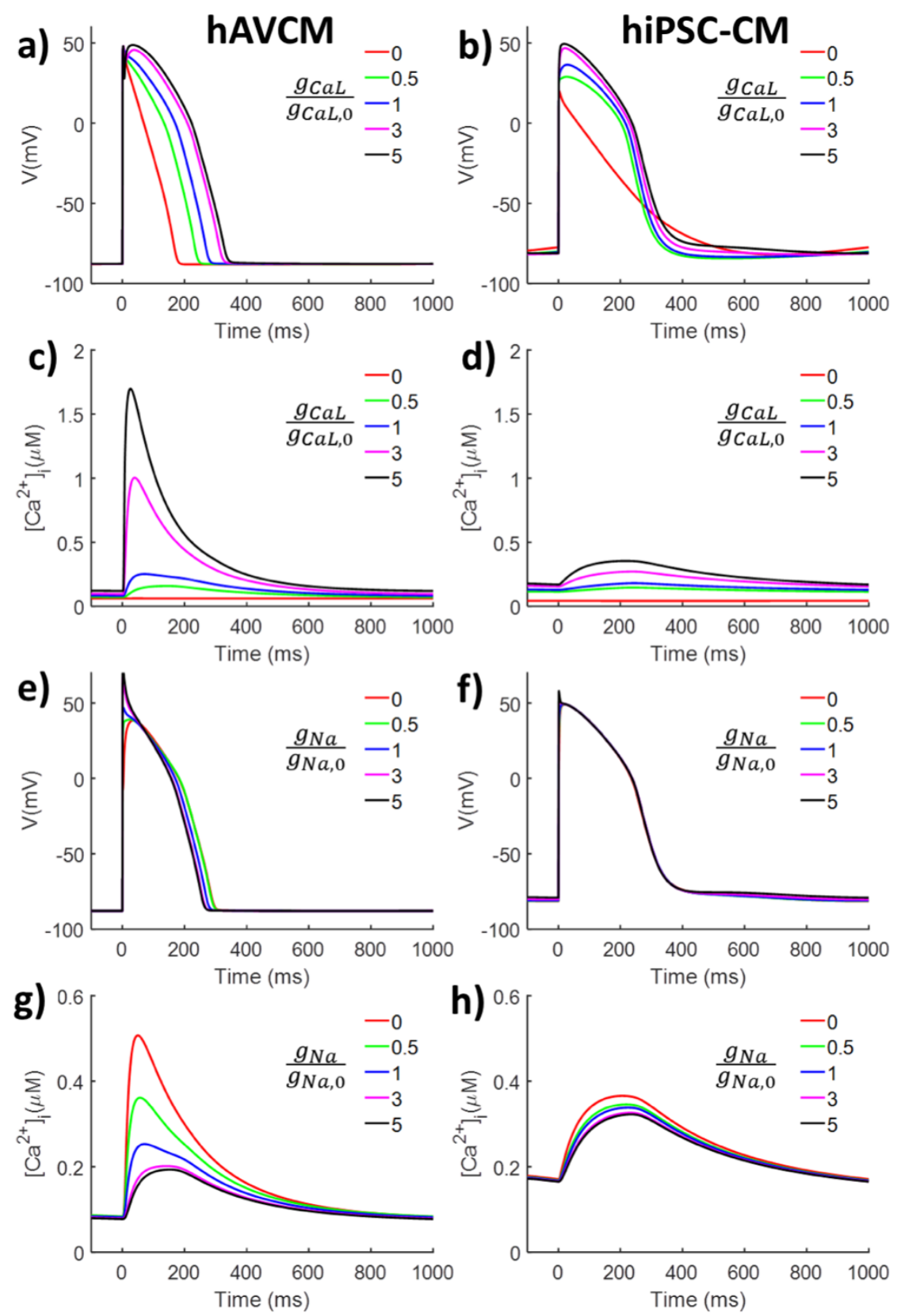

Figure 4. Effects of changing L-type $\mathrm{Ca}^{2+}$ channel conductance $\left(g_{C a L} / g_{C a L, 0}\right)$ on simulated action potential profiles for an hAVCM (a) and hiPSC-CM (iCell1) (b) and transients of internal calcium concentration $\left(\left[\mathrm{Ca}^{2+}\right]_{\mathrm{i}}\right)$ in $(\mathbf{c})$, (d) for an hAVCM and hiPSC-CM, respectively. Changing fast $\mathrm{Na}^{+}$channel conductance $\left(g_{N a} / g_{N a, 0}\right)$ had limited effects on the simulated action potential of hAVCM (e) and hiPSC-CM (f) as well as on calcium transients of hiPSC-CM (h). Changing fast $\mathrm{Na}^{+}$channel conductance $\left(g_{N a} / g_{N a, 0}\right)$ significantly changed peaks of calcium transients of $\operatorname{hAVCM}(\mathbf{g})$. 

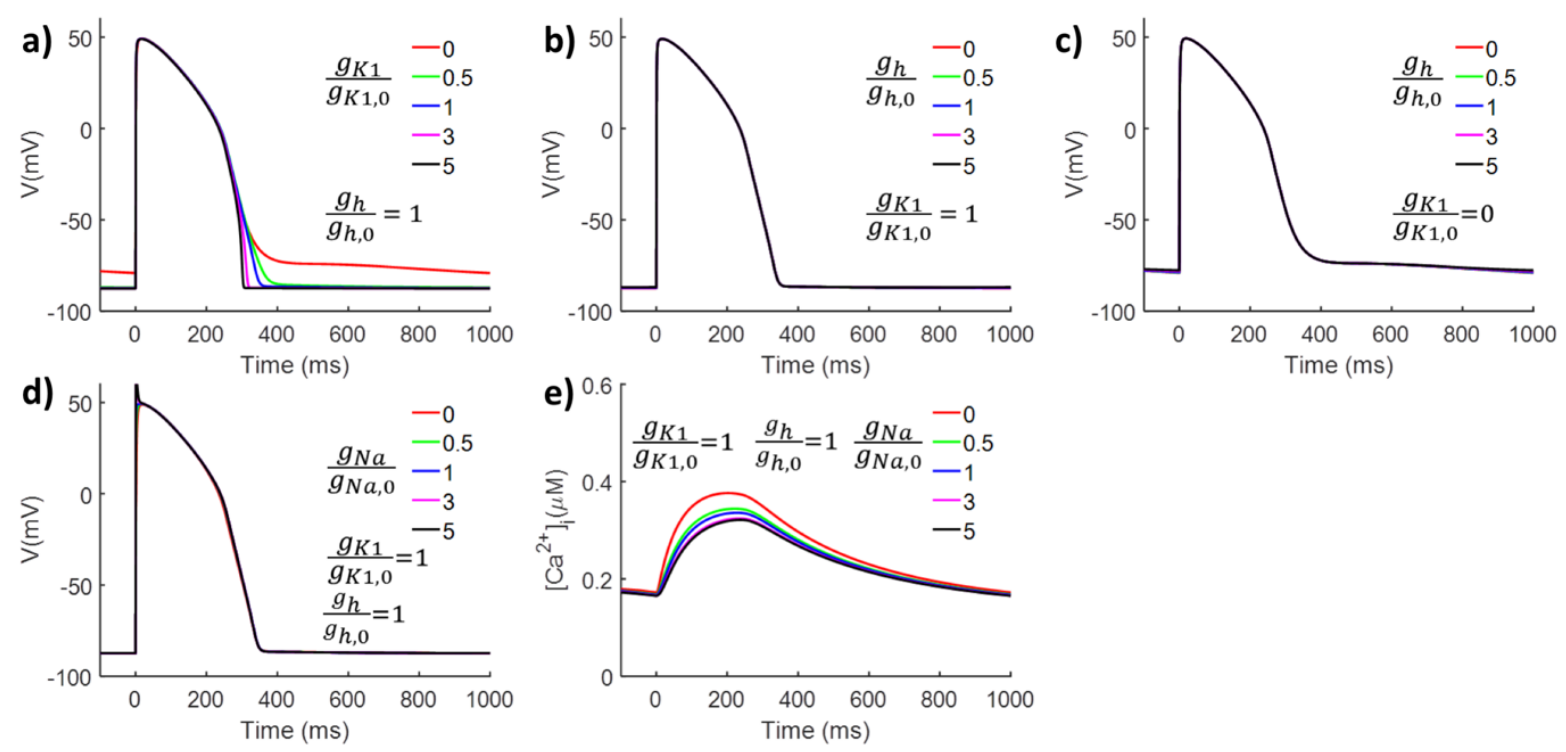

Figure 5. Influence of inward rectifier $\mathrm{K}^{+}$and hyperpolarization-activated $\mathrm{Na}^{+} / \mathrm{K}^{+}$channel conductance on the iCell1 version of the hiPSC-CM model. While keeping conductances of hyperpolarization-activated $\mathrm{Na}^{+} / \mathrm{K}^{+}$channel, $g_{h}$, at the level of hAVCM, increasing conductance of inward rectifier $\mathrm{K}^{+}$channel, $g_{K 1}$, lowered the resting membrane potential to $\sim-90 \mathrm{mV}(\mathbf{a})$. If $g_{K 1}$ was kept at the level of hAVCM, changing, $g_{h}$, had no effects on action potential profiles (b). If $g_{K 1}$ is low, changing $g_{h}$ had no effects to lower the resting membrane potential (c). When $g_{K 1}$ and $g_{h}$ are at the level of hAVCM, effects of changing fast $\mathrm{Na}^{+}$channel conductance, $g_{N a}$, on action potential profiles (d) and calcium transients (e) became similar to those of hAVCM (Figure $4 \mathbf{e}$ and $\mathbf{g}$ ). 

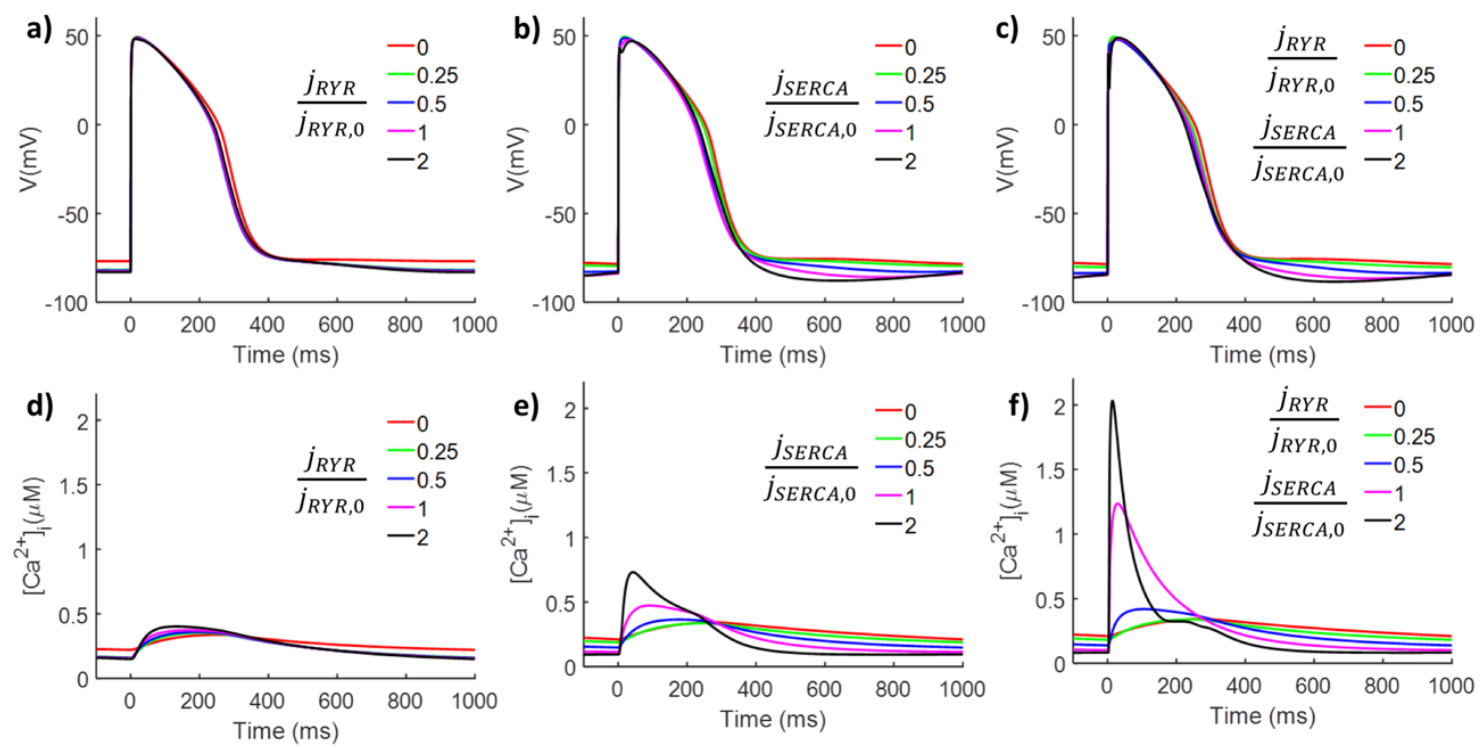

Figure 6. Effects of changing hiPSC-CM's (iCell1) $\mathrm{Ca}^{2+}$ release via ryanodine receptors, $j_{R Y R}$, and $\mathrm{Ca}^{2+}$ uptake via SERCA pump, $j_{S E R C A}$, on the simulated action potential profiles $(\mathbf{a}, \mathbf{b})$ and calcium transients $(\mathbf{d}, \mathbf{e})$. Increasing $j_{R Y R}$ and $j_{S E R C A}$ at the same time to the level of hAVCM reduced resting membrane potentials (c) and increased calcium transient's amplitudes (f). 


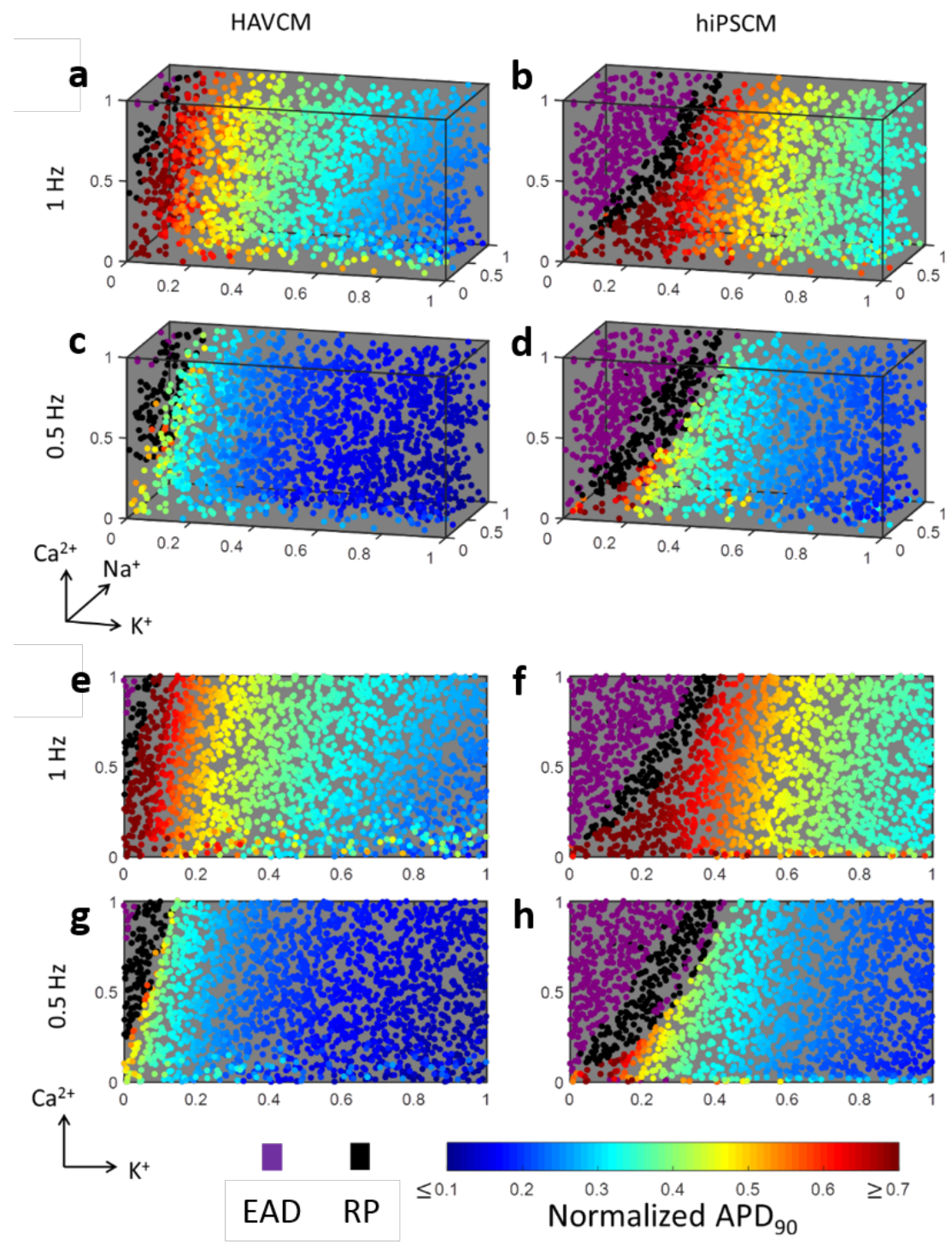

Figure 7. Action potential durations at $90 \%$ from their peak $\left(\mathrm{APD}_{90}\right)$ were normalized by pacing period (i.e., $1 \mathrm{~s}$ and $2 \mathrm{~s}$ for 1 and $0.5 \mathrm{~Hz}$ pacing frequency, respectively) and color-coded (blue red). Proarrhythmic action potential profiles were categorized into those exhibiting early after depolarization (EAD, purple) and repeating oscillation (RP, black). Combined effects of inhibiting conductance of $\mathrm{K}^{+}, \mathrm{Na}^{+}$, and $\mathrm{Ca}^{2+}$ channels at various degrees were plotted in 3D space with the color code for hAVCM (a) and hiPSC-CM (iCell1) (b) that were electrically paced at $1 \mathrm{~Hz}$. Decreasing pacing frequency to $0.5 \mathrm{~Hz}$ enlarged a region exhibiting EAD and RP in hAVCM (c) and hiPSC-CM (d). 2D scatter plots of hAVCM (e and $\mathbf{g}$ ) and hiPSC-CM (f and $\mathbf{h}$ ) were generated by projecting color-coded dots on to a $\mathrm{K}^{+}-\mathrm{Ca}^{2+}$ plane. Latin hypercube sampling was used to select combinations of $\mathrm{K}^{+}, \mathrm{Na}^{+}$, and $\mathrm{Ca}^{2+}$ inhibition. 


\section{References}

1 Laverty, H. G. et al. How can we improve our understanding of cardiovascular safety liabilities to develop safer medicines? British Journal of Pharmacology 163, 675-693, (2011).

2 de Abajo, F. J. \& Rodriguez, L. A. G. Risk of ventricular arrhythmias associated with nonsedating antihistamine drugs. Brit J Clin Pharmaco 47, 307-313, (1999).

3 Koerner, J. E. \& Seigl, P. K. S. in Global Approach in Safety Testing Vol. 5 AAPS Advances in the Pharmaceutical Sciences (eds J. W. van der Laan \& J. J. DeGeorge) Ch. 11, 243-265 (Springer, 2013).

4 Stockbridge, N., Morganroth, J., Shah, R. R. \& Garnett, C. Dealing with global safety issues: was the response to QT-liability of non-cardiac drugs well coordinated? Drug safety 36, 167-182, (2013).

5 Gintant, G. An evaluation of hERG current assay performance: Translating preclinical safety studies to clinical QT prolongation. Pharmacol Ther 129, 109-119, (2011).

6 Colatsky, T. et al. The Comprehensive in Vitro Proarrhythmia Assay (CiPA) initiative. Update on progress. J Pharmacol Toxicol Methods 81, 15-20, (2016).

7 Huo, J. et al. Evaluation of batch variations in induced pluripotent stem cell-derived human cardiomyocytes from 2 major suppliers. Toxicological Science 156, 25-38, (2017).

8 Doss, M. X. et al. Maximum diastolic potential of human induced pluripotent stem cellderived cardiomyocytes depends critically on $\mathrm{I}_{\mathrm{Kr}}$. PLoS One 7, (2012).

9 Chi, K. R. Revolution dawning in cardiotoxicity testing. Nat Rev Drug Discov 12, 564-566, (2013).

10 He, J. Q., Ma, Y., Lee, Y., Thomson, J. A. \& Kamp, T. J. Human embryonic stem cells develop into multiple types of cardiac myocytes: action potential characterization. Circulation Research 93, 32-39, (2003).

11 Lieu, D. K. et al. Mechanism-based facilitated maturation of human pluripotent stem cellderived cardiomyocytes. Circ Arrhythm Electrophysiol 6, 191-201, (2013).

12 Paci, M., Hyttinen, J., Aalto-Setala, K. \& Severi, S. Computational models of ventricularand atrial-like human induced pluripotent stem cell derived cardiomyocytes. Annals of Biomedical Engineering 41, 2334-2348, (2013).

13 Ma, J. Y. et al. High purity human-induced pluripotent stem cell-derived cardiomyocytes: electrophysiological properties of action potentials and ionic currents. American Journal of Physiology-Heart and Circulatory Physiology 301, H2006-H2017, (2011).

14 O'Hara, T., Virag, L., Varro, A. \& Rudy, Y. Simulation of the undiseased human cardiac ventricular action potential: model formulation and experimental validation. PLoS Comput Biol 7, e1002061, (2011).

15 Lopez-Izquierdo, A. et al. A near-infrared fluorescent voltage-sensitive dye allows for moderate-throughput electrophysiological analyses of human induced pluripotent stem cellderived cardiomyocytes. American Journal of Physiology-Heart and Circulatory Physiology 307, H1370-H1377, (2014).

16 Hoekstra, M., Mummery, C. L., Wilde, A. A. M., Bezzina, C. R. \& Verkerk, A. O. Induced pluripotent stem cell derived cardiomyocytes as models for cardiac arrhythrnias. Front Physiol 3, 346, (2012). 
17 Bett, G. C. L. et al. Electronic "expression" of the inward rectifier in cardiocytes derived from human-induced pluripotent stem cells. Heart Rhythm 10, 1903-1910, (2013).

18 Louch, W. E., Koivumaki, J. T. \& Tavi, P. Calcium signalling in developing cardiomyocytes: implications for model systems and disease. J Physiol 593, 1047-1063, (2015).

19 Gherghiceanu, M. et al. Cardiomyocytes derived from human embryonic and induced pluripotent stem cells: comparative ultrastructure. J Cell Mol Med 15, 2539-2551, (2011).

20 Krogh-Madsen, T., Sobie, E. A. \& Christini, D. J. Improving cardiomyocyte model fidelity and utility via dynamic electrophysiology protocols and optimization algorithms. $J$ Physiol 594, 2525-2536, (2016).

21 Livshitz, L. M. \& Rudy, Y. Regulation of $\mathrm{Ca}^{2+}$ and electrical alternans in cardiac myocytes: role of CAMKII and repolarizing currents. American Journal of Physiology-Heart and Circulatory Physiology 292, H2854-H2866, (2007).

22 Gintant, G., Sager, P. T.\& Stockbridge, N. Evolution of strategies to improve preclinical cardiac safety testing. Nat Rev Drug Discov 15, 457-471, (2016).

23 Mays, D. J., Foose, J. M., Philipson, L. H. \& Tamkun, M. M. Localization of the Kv1.5 K channel protein in explanted cardiac tissue. Journal of Clinical Investigation 96, 282-292, (1995).

24 Amos, G. J. et al. Differences between outward currents of human atrial, and subepicardial ventricular myocytes. J Physiol 491, 31-50, (1996).

25 Courtemanche, M., Ramirez, R. J. \& Nattel, S. Ionic mechanisms underlying human atrial action potential properties: insights from a mathematical model. American Journal of Physiology-Heart and Circulatory Physiology 275, H301-H321, (1998).

26 Kurata, Y., Matsuda, H., Hisatome, I. \& Shibamoto, T. Effects of pacemaker currents on creation and modulation of human ventricular pacemaker: theoretical study with application to biological pacemaker engineering. American Journal of Physiology-Heart and Circulatory Physiology 292, H701-H718, (2007).

27 Moroni, A. et al. Kinetic and ionic properties of the human HCN2 pacemaker channel. Pflugers Archiv-European Journal of Physiology 439, 618-626, (2000).

28 Herrmann, S., Layh, B. \& Ludwig, A. Novel insights into the distribution of cardiac HCN channels: an expression study in the mouse heart. Journal of Molecular and Cellular Cardiology 51, 997-1006, (2011).

29 Herrmann, S., Schnorr, S. \& Ludwig, A. HCN channels - Modulators of cardiac and neuronal excitability. Int J Mol Sci 16, 1429-1447, (2015).

30 Sartiani, L. et al. Developmental changes in cardiomyocytes differentiated from human embryonic stem cells: a molecular and electrophysiological approach. Stem Cells 25, 11361144, (2007). 


\title{
Supplement for "Computational Model for Simulating Drug-Induced Arrhythmia Sensitiv- ity of Human iPSC-derived Cardiomyocytes"
}

\author{
By Xin Gao ${ }^{2}$, Yue Yin ${ }^{1}$, Neil Daily ${ }^{1}$, Tyler Engel ${ }^{1}$, Li Pang ${ }^{3}$, Brian E. Carlson ${ }^{2}$ and Tetsuro Wakatsuki ${ }^{1}$
}

${ }^{1}$ InvivoSciences, Inc., Madison, WI

${ }^{2}$ Department of Molecular and Integrative Physiology, University of Michigan, Ann Arbor, MI

${ }^{3}$ Division of Biochemical Toxicology, National Center for Toxicological Research/FDA, Jefferson, AR

\section{Determining $\mathrm{dV} / \mathrm{dt}^{\mathrm{max}}$ from simulation results}

When evaluating the slope of the voltage upstroke at the beginning of the action potential $\left(\mathrm{dV} / \mathrm{dt}^{\max }\right)$ from simulation data, the value is highly dependent on the resolution of the time step selected. This means that when looking at experimental measurements of $\mathrm{dV} / \mathrm{dt}^{\max }$ the value can also vary depending on sampling rate. The table below shows at $1 \mathrm{~Hz}$ a $19 \%$ to $88 \%$ increase in the calculated $\mathrm{dV} / \mathrm{dt}^{\mathrm{max}}$ when dividing the time from stimulation to peak voltage over 500 intervals to find the largest value as opposed to 50 intervals. It is often not clear what the sampling rate is for $\mathrm{dV} / \mathrm{dt}^{\max }$ obtained experimentally so we assumed the upstroke was divided into 100 intervals in this study yielding values of $342 \mathrm{mV} / \mathrm{ms}$ for the hAVCM and $134 \mathrm{mV} / \mathrm{ms}$ for iCell1.

Table S1: Comparison of model predicted $(\mathrm{dV} / \mathrm{dt})_{\max }$.

\begin{tabular}{ccccccccc}
\hline \multirow{2}{*}{$\begin{array}{c}\text { Frequency } \\
(\mathbf{H z})\end{array}$} & $\begin{array}{c}\text { Sampling } \\
\text { Number } \\
(\mathbf{N})^{*}\end{array}$ & Cor4U1 & Cor4U2 & Cor4U3 & hAVCM & iCell1 & iCell2 & iCell3 \\
\cline { 3 - 9 } & 50 & 96 & 88 & 103 & 297 & 102 & 100 & 109 \\
\hline \multirow{2}{*}{1.0} & 100 & 112 & 121 & 114 & 342 & 134 & 120 & 153 \\
& 300 & 149 & 144 & 129 & 354 & 167 & 166 & 198 \\
& 500 & 154 & 146 & 130 & 354 & 172 & 174 & 206 \\
\hline 0.5 & 50 & 83 & 82 & 82 & 311 & 84 & 84 & 84 \\
& 100 & 86 & 84 & 84 & 342 & 88 & 87 & 89 \\
& 300 & 89 & 87 & 85 & 355 & 93 & 92 & 97 \\
& 500 & 89 & 87 & 85 & 356 & 94 & 93 & 98 \\
\hline
\end{tabular}

* Dividing the upstroke phase of action potential into $\mathrm{N}$ equal intervals to calculate $\mathrm{dV} / \mathrm{dt}$.

Supplemental Table S1. Variation in calculated $\mathrm{dV} / \mathrm{dt}^{\max }$ from simulation as a function of how the time from AP initiation to peak is divided. More time divisions (higher sampling number) capture the steepest section of the AP upstroke without averaging it over less steep regions. $\mathrm{dV} / \mathrm{dt}^{\mathrm{max}}$ measures obtained experimentally appear to be sampled at rates corresponding to a sampling number of 50-100 in this table. 


\section{Variation of $\mathrm{APD}_{90}$ with respect to fast $\mathrm{Na}^{+}$block for iCell1}

To show how invariant $\mathrm{APD}_{90}$ is to $\mathrm{Na}^{+}$block we took slices spanning a 0.1 range of $\mathrm{K}^{+}$block of our $\mathrm{Na}^{+} / \mathrm{K}^{+} / \mathrm{Ca}^{2+}$ blocking simulations for both hAVCM and hiPSC-CM (iCell1) at $1 \mathrm{~Hz}$ and $0.5 \mathrm{~Hz}$. All simulations contained within that slice are then projected onto the $\mathrm{Na}^{+} / \mathrm{Ca}^{2+}$ block plane to render the images below. It can be seen that at horizontal lines (constant $\mathrm{Ca}^{2+}$ and varying $\mathrm{Na}^{+}$block) across each one of these slices shows little variation in color indicating little change in $\mathrm{APD}_{90}$. This is to be expected in the hiPSC-CMs since in each hiPSC-CM model simulated the resting membrane potential is sightly depoarized $(-80$ to $-70 \mathrm{mV})$ preventing the inactivation gate of the fast $\mathrm{Na}^{+}$channel from resetting so block will have little effect on AP shape and $\mathrm{APD}_{90}$. In addition, the expression of fast $\mathrm{Na}+(\mathrm{SCN} 5 \mathrm{~A})$ is reduced to 0.65 of the expression of the hAVCM further limiting the effects of a fast $\mathrm{Na}^{+}$block. Even though the variation is larger in hAVCMs for fast $\mathrm{Na}^{+}$block it still shows a minimal contribution compared to L-type $\mathrm{Ca}^{2+}$ block.
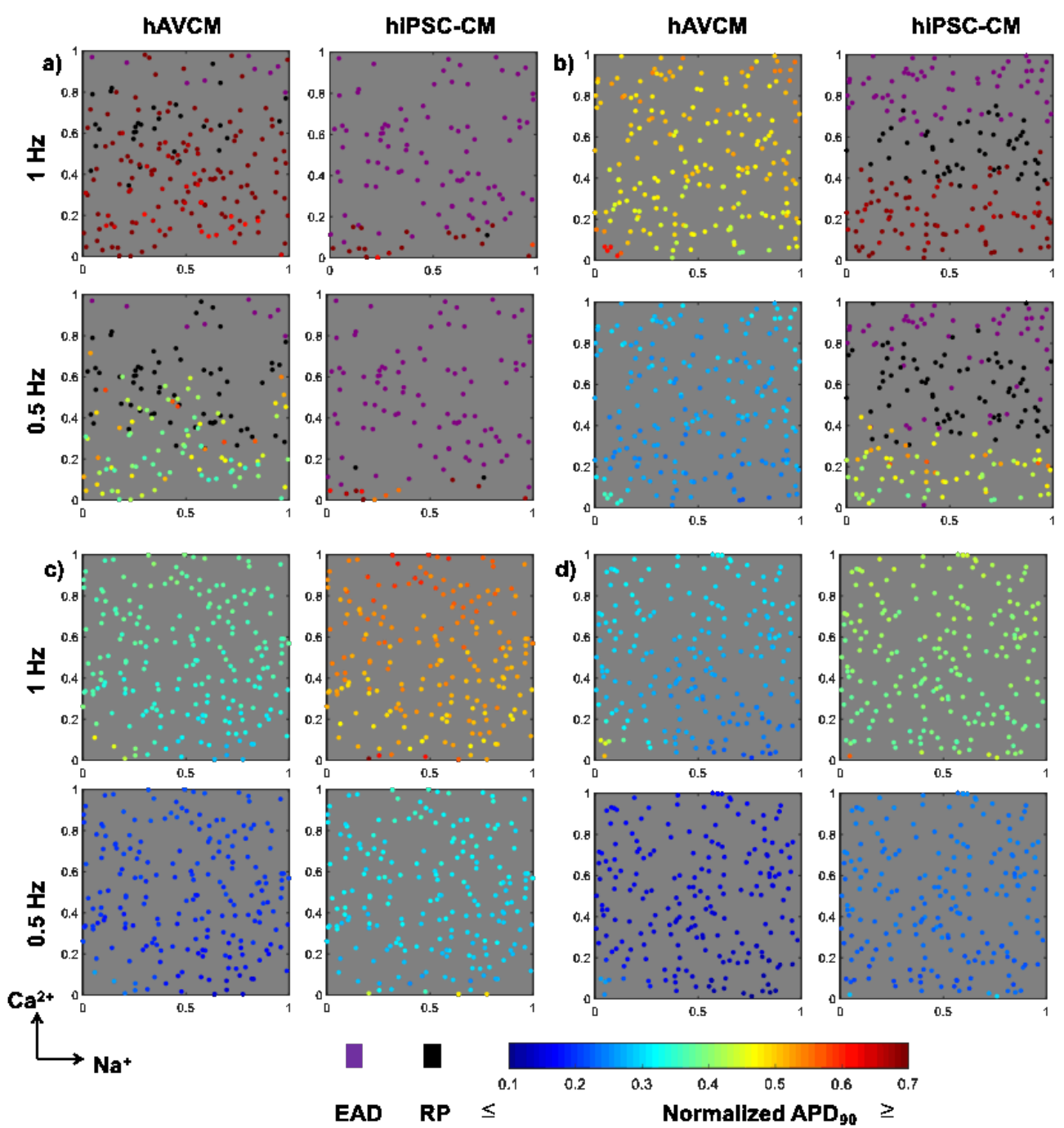

Supplement Figure S1.2D scatter plots by projecting color-coded dots of 3D scatter plots in Figure 7a-d to $\mathrm{Na}^{+} / \mathrm{Ca}^{2+}$ planes with the $\mathrm{K}^{+}$axis ranging from a) $\left.\left.0-0.1, \mathrm{~b}\right) 0.2-0.3, \mathrm{c}\right) 0.45-0.55$ and d) $0.7-0.8$. 


\section{Example of expression based scaling of ion channel, exchanger, pump and receptor function}

Each of the ion channels, exchangers, pumps and receptors for which expression data was obtained are modified to reflect the relative expression ratio with respect to the expression found in the hAVCM line. The modification assumes that the kinetic parameters and single component function remains the same and expression solely reflects the relative number of fully functioning ion channels, transporters, etc. This means that in the formulation of an ion channel we are purely scaling the maximal conductance parameter by the expression ratio. The maximal conductance represents the conductance if all channels of that particular type on the cell membrane were open. Maximal conductance parameters were scaled for the: fast $\mathrm{Na}^{+}$channel, transient outward $\mathrm{K}^{+}$channel, rapid delayed rectifier $\mathrm{K}^{+}$channel, slow delayed rectifier $\mathrm{K}^{+}$ channel, inward rectifier $\mathrm{K}^{+}$channel, $\mathrm{Na}^{+} / \mathrm{Ca}^{2+}$ exchanger, hyperpolarization activated $\mathrm{Na}^{+} / \mathrm{K}^{+}$channel and ultrarapid $\mathrm{K}^{+}$channel. For the L-type $\mathrm{Ca}^{2+}$ channel the maximal permeability was scaled and for the ryanodine receptor and sarco/endoplasmic reticulum $\mathrm{Ca}^{2+}$ ATPase maximal fluxes were scaled.

As an example of how we altered the original ORd formulation, the complete formulation for the delayed rectifier $\mathrm{K}^{+}$channel is given below with the modification due to expression data in bold font:

$$
\begin{aligned}
& x_{r, \infty}=\frac{1}{1+e^{-(V+8.337) / 6.789}} \\
& \tau_{x r, f}=12.98+\frac{1}{0.3652 \cdot e^{(V-31.66) / 3.869}}+0.00004123 \cdot e^{-(V-47.78) / 20.38} \\
& \tau_{x r, s}=1.865+\frac{1}{0.06629 \cdot e^{(V-34.70) / 7.355}}+0.00001125 \cdot e^{-(V-29.74) / 25.94} \\
& \frac{d x_{r, f}}{d t}=\frac{x_{r, \infty}-x_{r, f}}{\tau_{x r, f}} \\
& \frac{d x_{r, s}}{d t}=\frac{x_{r, \infty}-x_{r, s}}{\tau_{x r, s}} \\
& x_{r}=\left[\frac{1}{1+e^{(V+54.81) / 38.21}}\right] x_{r, f}+\left[1-\frac{1}{1+e^{(V+54.81) / 38.21}}\right] x_{r, s} \\
& R_{K r}=\left[\frac{1}{1+e^{(V+55.0) / 75.0}}\right] \cdot\left[\frac{1}{1+e^{(V-10.0) / 30.0}}\right] \\
& I_{K r}=I_{K r, E x p r} \cdot g_{K r} \cdot \sqrt{\frac{K}{5.4}} \cdot x_{r} \cdot R_{K r} \cdot\left(V-E_{K}\right) \\
&
\end{aligned}
$$

Where $x_{r, \infty}$ is the steady state open probability of the activation gate, $V$ is the membrane potential, $\tau_{x r . f}$ is the time constant of the fast activation component of the activation gate, $\tau_{x r, s}$ is the time constant of the slow deactivation component of the activation gate, $x_{r}$ is the open probability of the activation gate, $R_{K r}$ is the instantaneous open probability of the inactivation gate, $I_{K r}$ is the current through the rapid delayed rectifier channel, $I_{K r, E x p r}$ is the ratio of the $\mathrm{KCNH} 2$ expression in the hiPSC-CM with respect to that measured 
in the hAVCM, $g_{K r}$ is the maximal rapid delayed rectifier conductance of an hAVCM, $K_{o}$ is the extracellular $\mathrm{K}^{+}$concentration and $E_{K}$ is the Nernst potential for $\mathrm{K}^{+}$.

In three of the channels (fast $\mathrm{Na}^{+}, \mathrm{L}-$ type $\mathrm{Ca}^{2+}$ and rapid delayed rectifier $\mathrm{K}^{+}$) blocking is achieved by adding an additional term to the equation for current. Therefore, in the case of the rapid delayed rectifier current given above $I_{K r}$ is given by:

$I_{K r}=\boldsymbol{I}_{\boldsymbol{K} \boldsymbol{r}, \boldsymbol{E x p r}} \cdot \boldsymbol{I}_{\boldsymbol{K r}, \boldsymbol{b l o c k}} \cdot g_{K r} \cdot \sqrt{\frac{K_{o}}{5.4}} \cdot x_{r} \cdot R_{K r} \cdot\left(V-E_{K}\right)$

where $I_{K r, b l o c k}$ is the fraction of total current flowing through the channel after block and goes from 0 (100\% block) to 1 (no block). 